\title{
Laboratory Markers and Characteristics of New HIV Infections Among Adolescents in Zambia
}

\author{
Tina Nanyangwe Moyo ( $\nabla$ tina.nanyangwe@pku.edu.cn ) \\ Peking University https://orcid.org/0000-0002-9175-323X \\ Nkuye Moyo \\ Peking UNiversity, Institute of Population Research \\ Xiaoying Zheng \\ Peking University, Institute of Population Research \\ Samuel Kalibala \\ Population Council, Washington DC Office \\ Xiaochun Qiao \\ Peking University, Institute of Population Research \\ Chao Guo \\ Peking University, Institute of Population Research \\ Kim Sweeny \\ Victoria Institute of Strategic Economic Studies, Victoria University \\ Jilei Wu \\ Peking University, Institute of Population Research
}

\section{Research article}

Keywords: HIV RNA, New HIV Infections, Adolescents, Young People, CD4+ T-cell Count, Viral Load, Hepatitis B, Syphilis

Posted Date: July 15th, 2020

DOI: https://doi.org/10.21203/rs.3.rs-42500/v1

License: (c) (i) This work is licensed under a Creative Commons Attribution 4.0 International License. Read Full License 


\section{Abstract \\ Background}

We aimed to explore HIV RNA (ribonucleic acid) virologic levels greater than 1,000 copies/millilitre (ml), among HIV-positive adolescents aged 15-24 years, establish the spread of CD4 T- cell counts and inspect characteristics of adolescents presenting with new HIV infections, including co-infections with Hepatitis B virus and syphilis.

\section{Methods}

We analysed data from the Zambia Population-based HIV Impact Assessment 2016 survey. Two-stage stratified cluster probability sample design was used to select the target population. Our study truncated the population to focus on the age-group 15-24 whose biomarker tests and household information were complete. Our primary outcome measure was "New HIV-positive Infections among 15-24-year-olds" defined as HIVpositive biomarker samples presenting with HIV RNA $\geq 1,000$ copies/ml without detectable ARVs. We tested associations between new HIV infections and clinical characteristics using negative binomial models adjusting for age, sex, education, marital-status, residence among several covariates.

\section{Results}

Overall, $2 \cdot 3 \%$ ([166/7320], 95\% Cl: 1.9-2.6) adolescents aged 15-24 years were diagnosed with new HIV infections, with greater proportions among females $(3 \cdot 3 \%$ [139/4,165], 95\% Cl: $2 \cdot 8-3 \cdot 9)$ than males ( $0 \cdot 86 \%$ [27/3,155], 95\% Cl: $0 \cdot 6-1 \cdot 2)$. Almost half (47.6\%) of seroconversions had HIV RNA $\geq 50,000$ copies/ml and an average CD 4 T-cell count of 479 cells $/ \mathrm{mm} 3$. HBV- positive adolescents (IRR 8.6, 95\% Cl: 2.9-24.9, P< 0.001, model 2) were at increased risk of new infections unlike those testing positive for syphilis antibodies (IRR 1.22, 95\% Cl: $0 \cdot 2-8 \cdot 3, \mathrm{P}<0 \cdot 84$ ). Adjusting for confounders revealed that being married or cohabiting, testing positive for $\mathrm{HBV}$, being a rural resident and attaining higher than secondary education emerged as strongest correlates of new infections.

\section{Conclusion}

High baseline levels of viral load and low CD4 + T-cell count in recent HIV infections among adolescents indicate weak immune repertoire at first diagnosis, increasing the risk of contagion. As the epidemic continues to spread within the adolescent population, HIV-infection will become more complex and greater proportions of adolescents will likely be infected by regular partners. This suggests growing need for interventions targeted at stable partnerships and intensified public health campaigns specific for adolescents.

\section{Background}

The human immunodeficiency virus (HIV) infection is one common cause of adolescent hospitalization because of the continuum of opportunistic infections and high burden of protracted complications due to HIV/AIDS (1). UNAIDS reveals that in 2015, 260000 [180 $000-340$ 000] new HIV infections were diagnosed among adolescents in sub-Saharan Africa, estimated at 29 adolescents acquiring HIV every hour (2). Estimates further indicate that young girls aged 15-19 accounted for almost $80 \%$ of these recent infections (2). The proportion of young people living with HIV rose globally by $30 \%$ between 2005 and 2016 and those dying due to AIDS-related illnesses tripled, making it the only age group to have experienced an increase (3). Adolescents' risk of acquiring HIV is closely correlated, among other factors, with age at sexual debut, low condom use, low counseling and testing coverage, legal and structural barriers $(2,4)$.

DIrrespective of achievements so far made in responding to HIV, approximately 60,000 persons were diagnosed with new HIV infections in Zambia in 2015 comprising 50,000 adults and 8,900 children $(2,5)$. Youth are the least likely than any other age group to be aware of their infection. For example, only $42 \%$ of adolescents (aged 15-24) in Zambia knew their HIV status compared to $67 \%$ among adults $15-59$ years in 2016 (6). Also, population-based survey data from sub-Saharan Africa shows that only $9 \%$ of young men 15-19 compared to $13 \%$ of of their girls contemporaries had tested and received results for HIV in the last 12 months $(7,8)$. Compounding the status quo are sex disproportions in the prevalence of HIV. Within the population aged $20-24$, females exhibit higher prevalence levels than males (5.6\% versus $1.8 \%$ ) (5). Adolescent girls who reported being divorced, separated, or widowed had higher (13.4\%) HIV prevalence than their currently married and never-married counterparts ( $6.2 \%$ and $4.8 \%$ respectively) (9). In spite of growing accessibility to effective HIV prevention tools, methods and substantial scaleup of HIV treatment, attainment of viral load suppression (VLS) remains distinctly low in the younger population: $34 \%$ in HIV-positive females and $35.7 \%$ among HIV-positive males aged 15 to 24 compared to $73.5 \%$ in HIV-positive women and $73 \%$ in HIV-positive men aged 45 to 59 years (10). This rate of progress towards slowing the incidence of HIV acquisition, bolstering accessibility to treatment, and stopping AIDS-related deaths particularly among adolescents demands expansion and scaled up in order to reach high-incidence locations and maximize impact (11). 
Because several clinical studies of HIV infection $(5,12-14)$, often disaggregate the population aged $0-14$ years as children and $15-49$ years as adults, in so doing, the probability of missing distinctive features of recent infections among a special group of adolescents aged $15-24$ years is high. The age range 15-24 years aligns with the concept that adolescence might best be considered as ranging from 10 to 24 years because the transition period from childhood to adulthood continues into the twenties (15), and occupies a substantive part of their life course and shifting patterns of health and wellbeing (16). For this reason, it has been proposed that when adolescence is perceived as the population of young people from 10 to 24 years (15), it would help create opportunities for adolescents to acquire valuable assets and capabilities relevant, among others benefits, to averting health risk (17). However, for this study, we consider young people aged 15-24 years as adolescents. Herein, we explore the HIV RNA (ribonucleic acid) virologic levels greater than 1,000 copies/millilitre (ml) with undetectable antiretrovirals (ARVs), among HIV-positive adolescents aged 15-24 years, determine the distribution of CD4 + T-cell counts and inspect characteristics of adolescents presenting with new HIV infections, including co-infections with Hepatitis B virus (HBV) and syphilis.

\section{Methods}

\section{Study Design and Sample}

The Zambia Population-based HIV Impact Assessment (ZAMPHIA) 2016 dataset, on which this study is based, was a cross-sectional survey, nationally representative as described in the ZAMPHIA 2016 report (5), the study utilized the two-stage stratified cluster sample method, a probability proportional to size and an equal probability method to select the target population. The total sampled households were 13,441 , comprising 5,205 eligible females and 4,337 eligible males aged 15-24. Among the eligible population aged 15-24 years, 88.1\% (4,585/5,205) of women and $80 \cdot 5 \%(3,491 / 4,337)$ of men completed the interview. The response rate for biomarker testing was $90.6 \%(4,716 / 5,205)$ for females and $90 \cdot 0 \%(3,903 / 4,337)$ for males. Therefore, the sample size for our study was 7,320 comprising 4,165 girls and 3,155 boys aged $15-$ 24 who undertook biomarker testing in the ZAMPHIA 2016 study.

\section{Field-based Biomarker Testing}

All results for field-based tests were provided to clients and those testing positive were referred for treatment. As detailed in the ZAMPHIA 2016 report (5), the following tests were conducted in the field.

HIV Home-Based Testing and Counselling (HBTC) - This was performed on all sampled households. First, a screening test using Determine ${ }^{\mathrm{TM}}$ HIV-1/2 (Abbott Molecular Inc., Des Plaines, Illinois, United States) was performed. Participants diagnosed with a non-reactive result were reported HIV-negative. A confirmatory test using Uni-Gold ${ }^{\mathrm{TM}}$ (Trinity Biotech, plc. Wicklow, Ireland) was conducted for participants with reactive test results (18).

Hepatitis B Testing - As with HBTC, testing for HBV was done in every sampled household on individuals of all ages. The serological hepatitis B surface antigen rapid diagnostic test, Determine ${ }^{\mathrm{TM}} \mathrm{HBeAg}$, was used to determine acute or chronic HBV infection (5).

Syphilis Testing - All sampled participants in the age range 15-59 were tested for syphilis on DPP Syphilis Screen and Confirm Assay (Chembio, Medford, NY) in order to concurrently detect antibodies against non-Treponemal and Treponema pallidum antigens. Further confirmatory test was done on SD BIOLINE Syphilis 3.0 (Abbott Molecular Inc., Chicago, Illinois, United States) (5).

\section{Laboratory-based Biomarker Tests}

The following tests were performed in reference laboratories in Lusaka and Ndola.

Viral Load (VL) Testing - This test, also known as the HIV-1 RNA, was done for only verified positives using the Roche COBAS AmpliPrep Instrument and COBAS TaqMan 48 Analyzer on COBAS AmpliPrep/COBAS TaqMan HIV-1 Test, v2.0 (Roche Molecular Diagnostics, Branchburg, New Jersey, United States) (5). For samples with insufficient volume of plasma, HIV-1VL was measured from dried blood spot with the use of Abbott RealTime HIV-1 Assay (Abbott Molecular, Wiesbaden, Germany) (5). Definite HIV-positive samples also received a CD4 + T-cell count test on the Pima ${ }^{\text {TM }}$ CD4 Analyzer (Abbott Molecular Inc., Chicago, Illinois, United States) (5). Additionally, 5.0\% of randomly selected HIV-negative samples underwent the CD4 + T-cell count test.

\section{Estimating New HIV Infections}

To facilitate estimation of new HIV infections, the ZAMPHIA made use of two laboratory-based testing algorithms. 1) HIV-1 LAg Avidity enzyme immunoassay (EIA) (Serodia Biosciences Corporation, Portland, Oregon, United States) and VL (see Fig. 1) HIV-1 LAg Avidity EIA, VL, and ARV detection (5). Samples for which the median normalized optical density (ODn) was $\leq 1.5$ were categorized as newly infected specimens precipitating $\mathrm{VL}$ testing. Following this investigation, outcomes of $\mathrm{VL}<1,000$ copies/ml were labelled as old infections and $\mathrm{VL} \geq 1,000 \mathrm{copies} / \mathrm{ml}$ - new infections (5). When the ARV-controlled algorithm was considered, samples with $V L \geq 1,000$ copies/ml in which the presence of ARVs was 
detected were also grouped as old infections. Conversely, samples for which VL $\geq 1,000$ copies/ml with absence or undetectable ARVs were then categorized as new infections $(5,19)$.

Figure 1. STARHS biomarker detection schema used in estimating new HIV infections

\title{
[Figure 1 Here]
}

\author{
Reproduced from FHI (20).
}

The serological testing algorithm for recent HIV seroconversion (STARHS) estimates the immunological response against the virus on the basis of certain HIV antibody concentration, proportion (BED), isotype or avidity $(20,21)$. The time lapse from the point of acquisition (when antibodies can be detected) to the cut-off value defining confirmed infection status, or the window period, should be definitively established which is fundamental to the STARHS assays' capacity to specify the rate of incidence for a given population $(20,22)$.

\section{Measures}

The study aimed to find evidence to answer the following questions; Among the population aged 15-24 years, what proportion of HIV-positive samples indicated HIV RNA $\geq 1,000$ copies/ml with undetectable ARVs? What proportion of HIV-positive specimens with HIV RNA $\geq 1,000$ copies/ml without detectable ARVs, tested positive for HBV and syphilis antibody test? What is the CD4 + T-cell count distribution of HIV-positive samples with HIV RNA $\geq 1,000$ copies/ml without detectable ARVs, for the population 15-24 years? The CD4 + T-Cell count profiles how the immune system is functioning. The higher the T-Cell count, the better. As HIV infection progresses, the number of T-cells falls. The standard range for CD4 + T-cells runs between 500 and 1,500 cells/ $\mathrm{mm}^{3}$ (Pantaleo \& Fauci, 2005). However, most opportunistic infections (Ols) are found among patients with CD4 counts $<200$ cells $/ \mathrm{mm}^{3}$ (23). To investigate other determinants of new HIV infection in adolescents, our study endeavoured to answer the following questions. What underlying factors are associated with new HIV infections in adolescents. (e.g. new HIV infections with HBV and/or active syphilis antibodies)? Are there differences in new infections in adolescents depending on their background characteristics? A priori, we expected associations between new HIV infections in the population and the predictor variables. Our primary outcome measure (recent HIV infections in the population 15-24) was therefore, regressed on the four main predictor variables (age, sex, positive syphilis test and positive HBV test) across all models.

\section{Analytic Variables}

\section{Outcome}

The outcome variable was "New HIV-positive Infections among 15-24-year-olds" defined as HIV-positive biomarker samples with HIV RNA $\geq$ 1,000 copies/ml without detectable ARVs". It was a count variable. The focus for analysis was on samples that tested positive with HIV RNA $\geq$ 1,000 copies/ml with undetectable ARVs as these samples were classified as new infections. HIV-1 RNA is the response marker for antiretroviral therapy (ART). HIV RNA tests measure the amount of HIV in the blood. A low RNA means a person is less likely to transmit HIV. A patient's preART RNA level and the extent of RNA reduction following commencement of ART offers predictive insight about the prospects of disease progression (24).

\section{Predictor}

Predictor variables were clustered into laboratory markers (CD4 + T-cell count distribution, testing positive for hepatitis B virus and syphilis antibody test) and; demographic parameters (age and sex). The two laboratory markers, presence of hepatitis B virus and syphilis, were appropriate for assessing co-infection in HIV-positive individuals. HIV-positive persons who are concurrently positive for hepatitis B 'e'-antigen ( $\mathrm{HBeAg}$ ) usually present with higher hepatitis B viral load and fail to respond to antiviral treatment as positively as those with $\mathrm{HBeAg-negative}$ hepatitis B (25). Similarly, syphilis demonstrates an adverse impact on HIV infection, often revealing increases in RNA and corresponding decreases in CD4 + T-cell counts during active syphilis infection. Thus, individuals with HIV-syphilis co-infection are at increased risk of neurological complications and treatment failure (26).

\section{Covariates}

The third set of variables we considered were socioeconomic covariates deemed as influential determinants of new infections. They included marital status, education [highest level achieved], residence [rural, urban], wealth index, HIV testing history, and awareness of one's HIV-positive status.

\section{Statistical Analysis}

We summarized incidence of new HIV infections and immunological profiles according to demographic and socioeconomic characteristics. The likelihood ratio chi-square $\chi^{2}$ test aimed at testing whether the model containing the full set of predictors fits significantly better than a null (intercept only) model.; differences between predictor variables were assessed using Pearson chi-square $\chi^{2}$ test of independence or Fisher's exact 
test for variables with an expected frequency of cells of five or less (e.g. education). Continuous predictors were analyzed with a Student's t test for normally distributed variables, and the Wilcoxon-Mann-Whitney test (for age) or the Kruskal-Wallis test (for wealth quintile) for variables that did not have normal distributions. Univariate and bivariate analysis of variables reports proportions and binomial exact output at $95 \%$ confidence intervals $(\mathrm{Cl})$.

The number of new HIV infections is a count variable and closely follows a poisson distribution. We modelled the number of recent infections among young people using negative binomial regression. The negative binomial relative to count models such as the poisson or zero-inflated models, was considered suitable because the outcome variable was over-dispersed and did not have so many zeros. We further used generalized estimating equations and robust standard errors to determine factors independently and mutually associated with new HIV infections. When exponentiated, negative binomial regression coefficients provide the ratio of expected count per unit increase in exposure, referred to as the incident rate ratio (IRR) (27). We started by examining independent associations of each predictor variable and covariate (one at a time) with the outcome variable - new HIV infections (model 1). In model 2, all predictors and covariates with a P-value of up to 0.2 in model 1 were included. Associations were adjusted for potential confounders: education, marital status, wealth quintile, awareness of HIV-positive status, residence (rural/urban), number of sex partners in past 12 months and history of HIV testing. Model 3 considered all variables that were statistically significant at $p \leq 0.05$ in model 2 . Probabilities for removal and entry of predictor variables into the models were set at $p$-values of 0.20 . IRRs at $95 \%$ confidence intervals are reported, and p-values less than 0.05 were considered statistically significant. All statistical analysis was done in STATA 14.2 software (28).

\section{Results}

Overall incidence of new HIV infections for adolescents was from the general survey 2·3\% ([166/7,320], 95\% Cl: 1·94-2·63). Infections were notably greater in females $(3 \cdot 34 \%, 95 \% \mathrm{Cl}: 2 \cdot 81-3 \cdot 90)$ compared to male adolescents $(0 \cdot 86 \%, 95 \% \mathrm{Cl}: 0 \cdot 56-1 \cdot 24)$.

Characteristics of Adolescents Testing Newly Positive for HIV

Table 1 below shows detailed characteristics of adolescents testing newly positive to HIV. More females (83.7\%, 95\% Cl: 77.2-89.0) than males (16.3\%, 95\% Cl: $11 \cdot 0-22 \cdot 8)$ and more older adolescents (66.3\%, 95\% Cl: 58.5-73.4) aged 20-24 compared to younger ones (33.7\%, 95\% Cl: 26.6-41.5) aged 15-19 were diagnosed with recent HIV infections. Socioeconomic characteristics indicate larger proportions of unmarried adolescents $(63.9 \%, 95 \% \mathrm{Cl}: 56 \cdot 0-71 \cdot 2)$ than married contemporaries $(36 \cdot 1 \%, 95 \% \mathrm{Cl}: 28 \cdot 8-43 \cdot 9)$ testing newly positive for HIV. New infections were observed to rise with wealth and education level. For instance, $28.0 \%$ (95\% Cl: $21 \cdot 3-35 \cdot 6)$ of participants in the highest wealth quintile compared to $7 \cdot 3 \%(95 \% \mathrm{Cl}: 0 \cdot 56-1 \cdot 24)$ from the lowest tested positive. While $31 \cdot 9 \%$ adolescents with primary education relative to $63.9 \%$ with secondary education had seroconverted. Behavioral attributes further show that two-thirds (67.6\%) of adolescents that reported having had one sexual partner in 12 months prior to the survey compared to $19.9 \%$ with no partner had acquired the virus. Of all newly infected adolescents, only $14.5 \%$ were aware of their positive status, a consequence of low levels of HIV testing among young people (29-31). 
Characteristics of Adolescents Testing Newly Positive for HIV

\begin{tabular}{|c|c|c|c|}
\hline Predictor & Total $(n=166)$ & Percentage of New HIV Infections & $95 \% \mathrm{Cl}$ \\
\hline \multicolumn{4}{|l|}{ Sex } \\
\hline Male & 27 & $16.3(27 / 166)$ & $11.0-22.8$ \\
\hline Female & 139 & $83.7(139 / 166)$ & $77.2-89.0$ \\
\hline \multicolumn{4}{|l|}{ Age } \\
\hline $15-19$ & 56 & $33.7(56 / 166)$ & $26.6-41.5$ \\
\hline $20-24$ & 110 & $66.3(110 / 166)$ & $58.5-73.4$ \\
\hline \multicolumn{4}{|l|}{ Residence } \\
\hline Rural & 70 & $42.2(70 / 166)$ & $34.6-50.0$ \\
\hline Urban & 96 & $57.8(96 / 166)$ & $49.9-65.4$ \\
\hline \multicolumn{4}{|l|}{ Marital status } \\
\hline Not married & 106 & $63.9(106 / 166)$ & $56.0-71.2$ \\
\hline Married & 60 & $36.1(60 / 166)$ & $28.8-43.9$ \\
\hline \multicolumn{4}{|l|}{ Wealth quintile } \\
\hline Lowest & 12 & $7.3(12 / 164)^{*}$ & $3.8-12.4$ \\
\hline Second & 22 & $13.4(22 / 164)$ & $8.6-19.6$ \\
\hline Middle & 36 & $22.0(36 / 164)$ & $15.9-29.1$ \\
\hline Fourth & 48 & $29.3(48 / 164)$ & $22.4-36.9$ \\
\hline Highest & 46 & $28.0(46 / 164)$ & $21.3-35.6$ \\
\hline \multicolumn{4}{|l|}{ Education level } \\
\hline No education & 2 & $1.2(2 / 166)$ & $0.15-4.3$ \\
\hline Primary & 53 & $31.9(53 / 166)$ & $24.9-39.6$ \\
\hline Secondary & 106 & $63.9(106 / 166)$ & $56.0-71.2$ \\
\hline Tertiary & 5 & $5.0(5 / 166)$ & $0.9-6.8$ \\
\hline \multicolumn{4}{|l|}{ Active syphilis } \\
\hline Positive & 12 & $7.2(12 / 166)$ & $3.8-12.3$ \\
\hline Negative & 154 & $92.8(154 / 166)$ & $87.7-96.2$ \\
\hline \multicolumn{4}{|c|}{ Hepatitis B virus } \\
\hline Positive & 11 & $6.6(11 / 166)$ & $3.3-11.5$ \\
\hline Negative & 155 & $93.4(155 / 166)$ & $88.4-96.6$ \\
\hline \multicolumn{4}{|c|}{ Number of sex partners in last 12 months } \\
\hline No partner & 30 & $19.9(30 / 151)^{\star *}$ & $13.8-27.1$ \\
\hline One partner & 102 & $67.6(102 / 151)$ & $59.5-74.9$ \\
\hline More than one & 19 & $12.6(19 / 151)$ & $7.7-18.9$ \\
\hline \multicolumn{4}{|c|}{ Ever tested for HIV } \\
\hline Yes & 132 & $79.5(131 / 166)$ & $71.9-84.5$ \\
\hline
\end{tabular}

* “Wealth quintile” had 164 total observations instead of 166 because of missing values

**"Number of sex partners in last 12 months" also had less observations (151) than the expected 166 due to missing values 


\begin{tabular}{|c|c|c|c|}
\hline Predictor & Total $(n=166)$ & Percentage of New HIV Infections & $95 \% \mathrm{Cl}$ \\
\hline No & 34 & $20.5(34 / 166)$ & $15.1-28.0$ \\
\hline \multicolumn{4}{|c|}{ Aware of HIV + status } \\
\hline Aware & 24 & $14.5(24 / 166)$ & $9.5-20.7$ \\
\hline Not aware & 142 & $85.5(142 / 166)$ & $79.2-90.5$ \\
\hline
\end{tabular}

Virologic Outcomes of Adolescent with New HIV Infections

Slightly more than one third of new infections (36·7\% [61/166], 95\% Cl: $29 \cdot 41-44 \cdot 57)$ had HIV RNA viral loads clustered in the 10,001-50,000 copies/ml viral load category (see Fig. 2a.). HIV RNA viral load above 50,000 copies/ml is strongly associated with the risk of transmission (32). Findings show that the mean HIV RNA viral load for new HIV infections in adolescents was 164,183 copies/ml (95\% Cl: $64,886-263,480$ copies/ml, Fig. 2b.), more than three times the 50,000 copies/ml threshold. Cumulatively, nearly half (47.6\% [79/166], 95\% Cl: 39·80-55·47) of newly infected adolescents were diagnosed with HIV RNA viral load above 50,000 copies/ml (see red reference line in Fig. 2b.).

Disproportionately high viral load levels beyond 500,000 copies/ml were detected only in younger females adolescents aged $15-19$ (1.81\% [3/166]), (Fig. 2c. and Fig. 2d.) suggesting a possibility of acute infection at time of first diagnosis. Dissimilar to the HIV incidence pattern, males had higher proportions (59.3\% [16/27], 95\% Cl: 38·8-77·61) of HIV RNA $\geq 50,000 \mathrm{copies} / \mathrm{ml}$ than females (45·3\% [63/139], 95\% Cl: 36.9-54·0). There was also an observed predisposition for slightly more (48.2\% [27/56], 95\% Cl: 34.6-62.0, Fig. 2d.) younger adolescents (15-19) to have had viral loads greater than 50,000 copies/ml relative to the older cohort 20-24 years (45·5\%; [50/110], 95\% Cl: 35·9-55·3]).

\section{Figure 2: Viral Load and CD4 T-Cell Count for New HIV Infection in Adolescents}

[Figure 2 Here]

\section{Figure 2a:}

\section{Figure 2b:}

*Red reference line (yline) indicates the cut-of at HIV RNA 50,000 copies/ml

\section{Figure 2c:}

\section{Figure 2d:}

Immunological Status among New HIV Seroconverts

Study results further show an average CD4 + T-cell count of 479 cells/mm3 (95\% Cl: 445-513·6, Figs. 2b., 2c., and 3.) among newly HIV infected persons. Close to two-thirds (62\% [103/166], 95\% Cl 54-20-69·46) had CD4 + T-cell count $\leq 500$ cells/mm3 signaling poor immune repertoire (see Fig. 3. and Table 2.). There was a marginal variation in proportions by sex among individuals presenting with CD $4+\mathrm{T}$-cell count $\leq 500$

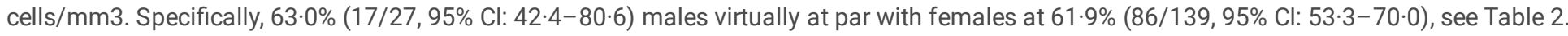

Figure 3. CD4 T-Cell Count of Newly Infected Adolescents

[Figure 3 Here]

${ }^{*}$ Red reference line (xline) shows CD4 + T-cell count cut-off at 500 cells $/ \mathrm{mm}^{3}$. The larger area on the left of the reference line reveals a greater proportion of adolescents presenting with decreased immunity at first diagnosis of a positive HIV serostatus.

On the other hand, age differentials revealed larger proportions (64.5\% [71/110], 95\% Cl: 54.9-73.4) of older adolescents ages $20-24$ years exhibiting diminishing immunological profiles of CD4 T- cell counts $\leq 500$ cells $/ \mathrm{mm}^{3}$ than younger adolescents $15-19$ years $(57 \cdot 1 \%$ [32/56], $95 \%$ $\mathrm{Cl} 43 \cdot 2-70 \cdot 3)$. Analysis to further understand the immunity status among seroconverts revealed that $29 \cdot 5 \%(7 / 139+34 / 139, \mathrm{Table} 2)$ females and $37 \%\left(7 / 27+3 / 27\right.$,) males had CD $4+$ T-cell count $\leq 350$ cells $/ \mathrm{mm}^{3}$. In addition, slightly more females $(38 \cdot 1 \%, 95 \%$ Cl: $30 \cdot 0-46 \cdot 7)$ presented with stronger immunity at CD4 + T-cell count greater than 500 cells $/ \mathrm{mm}^{3}$ compared to males (37.0\%, 95\% Cl: 19·4-57·6) males. Table 2 below illustrates further details of immunological statuses of adolescents by different characteristics. 
Table 2

Percentages of CD4 + T-Cell Count Levels for all Predictor Variables

\begin{tabular}{|c|c|c|c|c|c|}
\hline Predictor & & $\begin{array}{l}\leq 200 \\
\text { cells } / \mathrm{mm}^{3}\end{array}$ & $\begin{array}{l}201-350 \\
\text { cells } / \mathrm{mm}^{3}\end{array}$ & $\begin{array}{l}351-500 \\
\text { cells } / \mathrm{mm}^{3}\end{array}$ & $\begin{array}{l}>500 \\
\text { cells } / \mathrm{mm}^{3}\end{array}$ \\
\hline Total & & $6.0(10 / 166)$ & $24.7(41 / 166)$ & $31.3(52 / 166)$ & $38.0(63 / 166)$ \\
\hline \multicolumn{6}{|l|}{ Sex } \\
\hline & Male & $11.2(3 / 27)$ & $25.9(7 / 27)$ & $25.9(7 / 27)$ & $37.0(10 / 27)$ \\
\hline & Female & $5.0(7 / 139)$ & $24.5(34 / 139)$ & $32.4(45 / 139)$ & $38.1(53 / 139)$ \\
\hline \multicolumn{6}{|l|}{ Age } \\
\hline & $15-19$ & $7.1(4 / 56)$ & $19.6(11 / 56)$ & $30.4(17 / 56)$ & $42.9(24 / 56)$ \\
\hline & $20-24$ & $5.5(6 / 110)$ & $27.3(30 / 110)$ & $31.8(35 / 110)$ & $35.5(39 / 110)$ \\
\hline \multicolumn{6}{|l|}{ Residence } \\
\hline & Rural & $5.7(4 / 70)$ & $25.7(18 / 70)$ & 31.4 (22/70) & $37.1(26 / 70)$ \\
\hline & Urban & $6.3(6 / 96)$ & $24.0(23 / 96)$ & $31.3(30 / 96)$ & $38.5(37 / 96)$ \\
\hline \multicolumn{6}{|c|}{ Marital status } \\
\hline & Not married & $6.6(7 / 106)$ & $18.9(20 / 106)$ & $36.8(39 / 106)$ & $37.7(40 / 106)$ \\
\hline & Married & $5.0(3 / 60)$ & $35.0(21 / 60)$ & $21.7(13 / 60)$ & $38.3(23 / 60)$ \\
\hline \multicolumn{6}{|c|}{ Wealth quintile } \\
\hline & Lowest & $8.3(1 / 12)$ & $33.3(4 / 12)$ & $8.3(1 / 12)$ & $50.0(6 / 12)$ \\
\hline & Second & $4.5(1 / 22)$ & $13.6(3 / 22)$ & $50.0(11 / 22)$ & $31.8(7 / 22)$ \\
\hline & Middle & $2.8(1 / 36)$ & $25.0(9 / 36)$ & $33.3(12 / 36)$ & $38.9(14 / 36)$ \\
\hline & Fourth & $6.3(3 / 48)$ & $35.4(17 / 48)$ & $22.9(11 / 48)$ & $35.4(17 / 48)$ \\
\hline & Highest & $8.7(4 / 46)$ & $17.4(8 / 46)$ & $37.0(17 / 46)$ & $37.0(17 / 46)$ \\
\hline \multicolumn{6}{|c|}{ Education level } \\
\hline & No education & $0.0(0 / 2)$ & $50.0(1 / 2)$ & $50.0(1 / 2)$ & $0.0(0 / 2)$ \\
\hline & Primary & $1.9(1 / 53)$ & $26.4(14 / 53)$ & $24.5(13 / 53)$ & $47.2(25 / 53)$ \\
\hline & Secondary & $8.5(9 / 106)$ & $23.6(25 / 106)$ & $34.0(36 / 106)$ & $34.0(36 / 106)$ \\
\hline & Tertiary & $0.0(0 / 5)$ & $20.0(1 / 5)$ & $40.0(2 / 5)$ & $40.0(2 / 5)$ \\
\hline \multicolumn{6}{|c|}{ Active Syphilis } \\
\hline & Positive & $0.0(0 / 12)$ & $25.0(3 / 12)$ & $33.3(4 / 12)$ & $41.7(5 / 12)$ \\
\hline & Negative & $6.6(10 / 154)$ & $24.7(38 / 154)$ & $31.2(48 / 154)$ & $37.7(58 / 154)$ \\
\hline \multicolumn{6}{|c|}{ Hepatitis B Virus } \\
\hline & Positive & $0.0(0 / 11)$ & $18.2(2 / 11)$ & $36.4(4 / 11)$ & $45.5(5 / 11)$ \\
\hline & Negative & $6.5(10 / 155)$ & $25.2(39 / 155)$ & $31.0(48 / 155)$ & $37.4(58 / 155)$ \\
\hline \multicolumn{6}{|c|}{$\begin{array}{l}\text { Number of sex partners in last } 12 \\
\text { months }\end{array}$} \\
\hline & No partner & $3.3(1 / 30)$ & $20.0(6 / 30)$ & $33.3(10 / 30)$ & $43.3(13 / 30)$ \\
\hline & One partner & $5.9(6 / 102)$ & $29.4(30 / 102)$ & $31.4(32 / 102)$ & $33.3(34 / 102)$ \\
\hline & $\begin{array}{l}\text { More than } \\
\text { one }\end{array}$ & $5.3(1 / 19)$ & $15.8(3 / 19)$ & 42.1 (8/19) & $36.8(7 / 19)$ \\
\hline
\end{tabular}




\begin{tabular}{|c|c|c|c|c|c|}
\hline Predictor & & $\begin{array}{l}\leq 200 \\
\text { cells } / \mathrm{mm}^{3}\end{array}$ & $\begin{array}{l}201-350 \\
\text { cells } / \mathrm{mm}^{3}\end{array}$ & $\begin{array}{l}351-500 \\
\text { cells } / \mathrm{mm}^{3}\end{array}$ & $\begin{array}{l}>500 \\
\text { cells } / \mathrm{mm}^{3}\end{array}$ \\
\hline & Yes & $5.3(7 / 132)$ & $27.3(36 / 132)$ & $31.8(42 / 132)$ & $35.6(47 / 132)$ \\
\hline & No & $8.8(3 / 34)$ & $14.7(5 / 34)$ & $29.4(10 / 34)$ & $47.1(16 / 34)$ \\
\hline \multicolumn{6}{|c|}{ Aware of HIV + status } \\
\hline & Aware & $12.5(3 / 24)$ & $20.8(5 / 24)$ & $33.3(8 / 24)$ & $33.3(8 / 24)$ \\
\hline & Not aware & $4.9(7 / 142)$ & $25.4(36 / 142)$ & $31.0(44 / 142)$ & $38.7(55 / 142)$ \\
\hline
\end{tabular}

The study considered two common co-infections in HIV-positive adolescents; syphilis and HBV. Granted that syphilis, HBV and HIV can be acquired in similar ways, notwithstanding that syphilis and HBV are more infectious, co-infection occurs frequently $(25,26)$. Among adolescents with recent HIV infections, 7·2\% (12/166), 95\% Cl: 3·8-12·3) and 6.6\% (11/166), 95\% Cl: 3·3-11·5, Table 1) were diagnosed with syphilis and HBV co-infections, respectively. The risk of being diagnosed with a recent HIV infection significantly increased (IRR: 8.56, 95\% Cl: 2.94-24.93, P< 0.001 , model 2) with a positive HBV test relative to those testing negative. To the contrary, in model 2 , results suggest a near equal likelihood of HIV incidence among young people presenting with a seropositive syphilis test (IRR:1.23, 95\% Cl: $0 \cdot 18-8 \cdot 28, \mathrm{P}=0.84$ ) compared with individuals testing negative, even though the statistic was not significant. We further observed that the two co-infections were uniquely detected in samples with HIV RNA above 10,000 copies/ml.

Knowledge of HIV-positive Status among Newly HIV Infected Adolescents

Further analysis shows that one in every five (19.3\% [32/166], 95\% Cl: 13.57-26.11) adolescents testing with recent HIV infections in the survey were already aware of their HIV seropositive status. In spite of this verity, laboratory diagnostics did not detect ARVs in their blood samples an indication they had not initiated antiretroviral treatment. Statistically significant associations $(=5.659 ; \mathrm{P}<0.02)$ between knowledge of one's HIVpositive status and age were also established. Older adolescents aged 20-24 years had greater awareness of their HIV-positive status (12.7\% [21/166]; $95 \% \mathrm{Cl} 8 \cdot 0-18 \cdot 7)$ than younger adolescents aged $15-19$ years $(1 \cdot 8 \%$ [3/166]; 95\% $\mathrm{Cl} 0 \cdot 37-5 \cdot 2)$ on awareness of an individual's HIVpositive status.

[Figure $4 a$ and $4 b$ Here]

Even if $85 \cdot 5 \%$ of participants did not know they had HIV, older adolescents were relatively less aware(38.7\% [89/166]; $95 \%$ Cl: $45 \cdot 7-61 \cdot 4)$ than younger adolescents (31.9\% [53/166]; $95 \%$ Cl: 24.9-39.6, Figs. 4a. and 4b.). Within age and sex categories, more younger males (88.9\% [24/27], 95\% Cl 70.84-97.65, Fig. 4a.) and younger females (84.9\% [118/139], 95\% Cl: 77.83-90.40) were unaware of their HIV-positive serostatus than their older ones. Besides sex, Figure:4b shows that adolescents in urban areas were less inclined to knowing they had the virus at $50 \%$ (83/166; $95 \% \mathrm{Cl}: 42 \cdot 2-57 \cdot 8)$ than those residing in rural areas (35.5\%, 95\% Cl: $28 \cdot 3-43 \cdot 3)$.

Model Estimation Results 
Table 3

Generalized Negative Binomial Regression Models for New HIV Infections in Adolescents

\begin{tabular}{|c|c|c|c|c|c|c|c|c|c|}
\hline \multirow{3}{*}{ Predictors } & \multicolumn{3}{|l|}{ Model $1^{\dagger}$} & \multicolumn{3}{|c|}{ Model $2^{+\dagger}$} & \multicolumn{3}{|l|}{ Model $3^{t+\dagger}$} \\
\hline & \multirow[b]{2}{*}{$\mathrm{IRR}^{\mathrm{a}}$} & \multirow[b]{2}{*}{$\begin{array}{l}\text { P- } \\
\text { Value }\end{array}$} & \multirow[b]{2}{*}{$95 \% \mathrm{Cl}$} & \multirow[b]{2}{*}{ IRR } & \multirow[b]{2}{*}{$\begin{array}{l}\text { P- } \\
\text { Value }\end{array}$} & \multirow[b]{2}{*}{$95 \% \mathrm{Cl}$} & \multirow[b]{2}{*}{ IRR } & \multirow[b]{2}{*}{$\begin{array}{l}\text { P- } \\
\text { Value }\end{array}$} & \multirow[b]{2}{*}{$95 \% \mathrm{Cl}$} \\
\hline & & & & & & & & & \\
\hline Age & $1 \cdot 00069$ & 0.000 & $\begin{array}{l}1.000- \\
1.001\end{array}$ & $1 \cdot 06343$ & $0 \cdot 369$ & $\begin{array}{l}0.930- \\
1.216\end{array}$ & 0.984041 & 0.837 & $\begin{array}{l}0 \cdot 844- \\
1 \cdot 147\end{array}$ \\
\hline \multicolumn{10}{|l|}{ Sex } \\
\hline Female & $2 \cdot 27921$ & 0.000 & $\begin{array}{l}2 \cdot 277- \\
2 \cdot 281\end{array}$ & $0 \cdot 39227$ & $0 \cdot 011$ & $\begin{array}{l}0.190- \\
0.809\end{array}$ & 0.486252 & 0.044 & $\begin{array}{l}0.241- \\
0.980\end{array}$ \\
\hline Male & Ref. $^{b}$ & .. & .. & Ref. & .. & .. & Ref. & & \\
\hline \multicolumn{10}{|c|}{ Active syphilis $^{c}$} \\
\hline Positive & $0 \cdot 292607$ & $0 \cdot 162$ & $\begin{array}{l}0.0522- \\
1.640\end{array}$ & $1 \cdot 21842$ & $0 \cdot 840$ & $\begin{array}{l}0 \cdot 179- \\
8 \cdot 283\end{array}$ & 0.795605 & 0.735 & $\begin{array}{l}0 \cdot 212- \\
2 \cdot 990\end{array}$ \\
\hline Negative & Ref. & .. &.. & Ref. & .. & .. & Ref. &.. &.. \\
\hline \multicolumn{10}{|c|}{ Hepatitis B status } \\
\hline Positive & $7 \cdot 66655$ & 0.018 & $\begin{array}{l}6 \cdot 594- \\
41 \cdot 371\end{array}$ & $8 \cdot 56390$ & 0.000 & $\begin{array}{l}2.942- \\
24.931\end{array}$ & $6 \cdot 86583$ & 0.007 & $\begin{array}{l}1 \cdot 702- \\
27 \cdot 695\end{array}$ \\
\hline Negative & Ref. &.. &.. & Ref. & .. & .. & Ref. & & \\
\hline \multicolumn{10}{|c|}{ Marital status } \\
\hline Married & $4 \cdot 07027$ & 0.000 & $\begin{array}{l}4.067- \\
4.073\end{array}$ & $3 \cdot 15757$ & 0.037 & $\begin{array}{l}1 \cdot 071- \\
9 \cdot 313\end{array}$ & $2 \cdot 76422$ & $0 \cdot 117$ & $\begin{array}{l}0 \cdot 774- \\
9 \cdot 869\end{array}$ \\
\hline Not married & Ref. &.. &.. & Ref. & & & Ref. & & \\
\hline \multicolumn{10}{|l|}{ Residence } \\
\hline Rural & $2 \cdot 37048$ & 0.000 & $\begin{array}{l}1 \cdot 524- \\
3.687\end{array}$ & $3 \cdot 89909$ & 0.001 & $\begin{array}{l}1 \cdot 762- \\
8.631\end{array}$ & $3 \cdot 10161$ & 0.003 & $\begin{array}{l}1 \cdot 488- \\
6 \cdot 465\end{array}$ \\
\hline Urban & Ref. &.. &.. & Ref. &.. &.. & Ref. & & \\
\hline \multicolumn{10}{|c|}{ Wealth quintile } \\
\hline Lowest & Ref. &.. &.. & Ref. & .. &.. & - & & \\
\hline Second & 0.59644 & $0 \cdot 182$ & $\begin{array}{l}0 \cdot 279- \\
1 \cdot 274\end{array}$ & $1 \cdot 01981$ & 0.982 & $\begin{array}{l}0 \cdot 194- \\
5 \cdot 366\end{array}$ & - & & \\
\hline
\end{tabular}

†Model 1 is a bivariate model unadjusted for other variables. It examines association between each predictor variable and the outcome variable.

††Model 2 is a full controlled model with robust standard errors (SEs) in which inclusion into the model for all variables and covariates was contingent on passing the 0.2 p-value inclusion criterion in model 1are included. Hence, the variable, aware of one's HIV status was excluded.

t††Model 3 with robust SEs estimation include only statistically significant covariates. The restriction is relaxed for the four main predictors. Three variables, wealth quintile, ever tested for HIV and aware of one's HIV-positive status are not included. 


\begin{tabular}{|c|c|c|c|c|c|c|c|c|c|}
\hline \multirow[b]{2}{*}{ Middle } & \multicolumn{3}{|l|}{ Model $1^{\dagger}$} & \multicolumn{3}{|c|}{ Model $2^{t+}$} & \multicolumn{3}{|l|}{ Model $3^{t+t}$} \\
\hline & $0 \cdot 24992$ & 0.002 & $\begin{array}{l}0.106- \\
0.589\end{array}$ & $0 \cdot 70233$ & 0.65 & $\begin{array}{l}0.153- \\
3.233\end{array}$ & - & & \\
\hline Fourth & 0.33639 & 0.003 & $\begin{array}{l}0.164- \\
0.690\end{array}$ & $1 \cdot 12396$ & 0.874 & $\begin{array}{l}0.264- \\
4 \cdot 779\end{array}$ & - & & \\
\hline Highest & $0 \cdot 31899$ & 0.002 & $\begin{array}{l}0.156- \\
0.654\end{array}$ & $2 \cdot 42966$ & 0.235 & $\begin{array}{l}0.562- \\
10 \cdot 506\end{array}$ & - & & \\
\hline \multicolumn{10}{|l|}{ Education } \\
\hline No education & Ref. & .. & .. & Ref. & .. & .. & Ref. & & \\
\hline Primary & $5 \cdot 14169$ & 0.027 & $\begin{array}{l}1.202- \\
21 \cdot 985\end{array}$ & $10 \cdot 3383$ & 0.001 & $\begin{array}{l}2 \cdot 681- \\
39 \cdot 859\end{array}$ & $5 \cdot 891361$ & 0.026 & $\begin{array}{l}1 \cdot 236- \\
28 \cdot 080\end{array}$ \\
\hline Secondary & $1 \cdot 74790$ & 0.442 & $\begin{array}{l}0.421- \\
7 \cdot 260\end{array}$ & $7 \cdot 35867$ & 0.004 & $\begin{array}{l}1.922- \\
28 \cdot 169\end{array}$ & $6 \cdot 285652$ & 0.014 & $\begin{array}{l}1 \cdot 449- \\
27 \cdot 263\end{array}$ \\
\hline Higher than secondary & $1 \cdot 40491$ & 0.671 & $\begin{array}{l}0.292- \\
6.761\end{array}$ & 8.99769 & 0.014 & $\begin{array}{l}1 \cdot 557- \\
51 \cdot 990\end{array}$ & $12 \cdot 84462$ & 0.006 & $\begin{array}{l}2 \cdot 091- \\
78.997\end{array}$ \\
\hline \multicolumn{10}{|c|}{$\begin{array}{l}\text { Number of sex partners in past } 12 \\
\text { months }\end{array}$} \\
\hline One sex partner & Ref. & .. & .. & Ref. & .. &.. & Ref. & & \\
\hline No sex partner & $0 \cdot 28871$ & 0.011 & $\begin{array}{l}-1.296- \\
0.164\end{array}$ & $0 \cdot 79571$ & 0.500 & $\begin{array}{l}0.409- \\
1.547\end{array}$ & 0.29033 & 0.477 & $\begin{array}{l}0 \cdot 362- \\
1 \cdot 608\end{array}$ \\
\hline More than one sex part. & $-2 \cdot 0569$ & 0.000 & $\begin{array}{l}-3.081- \\
1 \cdot 0326\end{array}$ & $0 \cdot 19566$ & 0.002 & $\begin{array}{l}0.068- \\
0.559\end{array}$ & $0 \cdot 20708$ & 0.001 & $\begin{array}{l}0.084- \\
0.511\end{array}$ \\
\hline \multicolumn{10}{|l|}{ Ever tested for HIV } \\
\hline Yes & $1 \cdot 56962$ & 0.055 & $\begin{array}{l}0.991- \\
2 \cdot 486\end{array}$ & $0 \cdot 95721$ & 0.915 & $\begin{array}{l}0.429- \\
2 \cdot 135\end{array}$ & - & & \\
\hline No & Ref. & .. & .. & Ref. & .. & .. & - & & \\
\hline \multicolumn{10}{|l|}{ Aware of one's HIV + status } \\
\hline Yes & $1 \cdot 25719$ & $0 \cdot 840$ & $\begin{array}{l}0 \cdot 137- \\
11 \cdot 542\end{array}$ & - & & & - & & \\
\hline No & Ref. &.. & .. & - & & & - & & \\
\hline \multicolumn{10}{|c|}{$\begin{array}{l}\text { †Model } 1 \text { is a bivariate model unadjusted for other variables. It examines association between each predictor variable and the outcome } \\
\text { variable. }\end{array}$} \\
\hline \multicolumn{10}{|c|}{$\begin{array}{l}\text { ††Model } 2 \text { is a full controlled model with robust standard errors (SEs) in which inclusion into the model for all variables and covariates was } \\
\text { contingent on passing the } 0.2 \text { p-value inclusion criterion in model 1are included. Hence, the variable, aware of one's HIV status was excluded. }\end{array}$} \\
\hline
\end{tabular}

1. a. IRR - These are the incidence rate ratios for the negative binomial regression, showing the rate at which new HIV infections occurred.

2. b. Ref - refers to the variable category used as reference in the specified regression model.

3. c. Active syphilis variable meant the result for the simultaneous presence of antibodies against non-Treponemal and Treponema pallidum antigens clinical test.

We found evidence that incidence of new HIV infections is significantly different between males and females. Increased likelihood of new HIV infection was significantly associated with adolescent girls than boys (IRR 2·30; 95\% Cl: 2·27-2·28), p<0.001; Table 2, model1).

Younger participants had lower relative risk of new infection than older participants (IRR 1.00; 95\% Cl: 1·00-1.001), p<0.001; Table 2, model1). However, associations between age and new HIV infections were completely attenuated when adjustment for covariates was performed (Table 2, model 2 and model 3). Further independent associations were seen in individuals with active syphilis infection (IRR 0.29; 95\% Cl: 0.05-1.64; model 1), individuals in lower brackets of the wealth quintile (IRR 0.59; $95 \% \mathrm{Cl}$ : $0 \cdot 28-1 \cdot 27$; model 1 ) and those with more than one sexual partner in past 12 months (IRR 0.26; 95\% Cl: 0.09-0.77; model 1). Although mainly consistent in showing decreased likelihood of incidence of HIV infection, but most of these associations did not attain statistical significance. 
High educational attainment was associated with high relative risk of new HIV infection by up to 12.8 times (95\% Cl: 2.09-79.00, $\mathrm{p}<0 \cdot 01$; model 3) among those with higher than secondary education compared to individuals with no education. Other socio-behavioural covariates strongly associated with elevated relative risk were; adolescents aware of their HIV-positive status (IRR 1·26; 95\% Cl: 0.014-11·54; model 1), individuals that had previously tested for HIV (IRR 1.57; 95\% Cl: 0.99-2.49; model 1), those reporting one sex partner in past 12 months (IRR 2.07 ; $95 \%$ Cl: 1.18-3.65; in all three models), those married or cohabiting (IRR 3.16; 95\% Cl: 1.07-9.31; model 2) and adolescents residing in rural areas (IRR 3.90; 95\% Cl: 1·76-8.63; model 2 and model 3). With an exception of the variables "aware of one's HIV + status" and "ever tested for HIV", all estimates indicate statistically significant associations.

\section{Discussion}

Population-specific studies on new HIV infections are of great public health importance because they offer policy makers and planners definitive insights into the prevalence of the disease burden otherwise shrouded in population aggregation. Studies like ours aid in fully describing the epidemic, monitoring transmission patterns and prioritizing HIV prevention efforts targeted to specific groups such as the adolescent population Recently infected people with acute HIV infections contribute disproportionately to HIV transmission. Identifying individuals with new infections could have important implications at individual and public health levels. Our analysis quantified the incidence of new HIV infections among adolescents aged 15-24 years relative to standardized virologic and immunological outcomes. We also examined clinical and a range of sociodemographic parameters to uncover characteristics evidently correlated with new infections. Our results highlight that new HIV infections in adolescents are associated with co-infections including testing positive for hepatitis B virus (IRR 8.6 [2.9-24.9]), being female (IRR 1.9 [1.60$2 \cdot 24]$ ) than male (IRR 0.37 [0.24-0.54]), and being younger, between 15-19 years (IRR 1.4 [0.46-4.21]), than older (20-24 years) at IRR 0.71 $(0 \cdot 24-2 \cdot 15)$. Controlling for socio-demographic factors shows that being married or cohabiting, having higher than secondary education, belonging to a higher than middle wealth quintile, and being a rural resident were associated with increased risk for HIV incidence. Among behavioral attributes, having previously tested for HIV and reporting one sex partner in the past 12 months were associated with increased likelihood for infection.

Our study was not short of limitations. Accuracy in estimating HIV incidence and acute HIV infection in cross-sectional studies is sharply queried for suboptimal performance. The EIA and BED assays used in this study in the determination of recent HIV infections are used only on HIV seropositive specimens. This method employed the Serologic Testing Algorithm for Recent HIV Seroconversion (STARHS). However, this methodology and serologic assays therein have previously shown to contain substantial limitations, including biological, epidemiological, and statistical confounders (33). Additionally, although the EIA and BED assays used in our study are widely known, acceptance and use of these assays has been intensely disputed for its tendency to overestimate incidence (20). Therefore, we, may have overestimated the incidence of new HIV infections in adolescents and resulting associations with other parameters. Although we controlled for socioeconomic factors that have previously been linked to HIV prevalence; it is possible that residual confounding remains for parameters not considered in model estimation. Future studies should consider expanding confounders to augment appropriateness in determining factors associated with new HIV infections in sub-population groups. Nevertheless, granting that our study focuses on the population 15 to 24 years, the method used for new HIV infection estimation draws its strength from global recommendations stating that tendencies in prevalence among adolescents 15 to 24 years be utilized as proxy measures for calculating HIV incidence $(21,34)$. This is because sexual debut in this age group is expected to be recent such that prevalence closely reflects recent infections.

During the first few weeks following HIV-1 seroconversion, HIV RNA viral load surges, which poses considerable risk of HIV transmission (35, 36). Our study results show that the mean HIV RNA was 164,183 copies/ml $(64,886-263,480$ copies/ml, Fig. $2 b$.), three times more than the 50,000 copies/ml HIV RNA limit in someone not taking treatment. Which suggests that newly infected adolescents, on average, had high HIV RNA levels at point of first diagnosis. There is strong evidence from research that disease progression is substantially escalated in patients with HIV-1 RNA levels > 100,000 copies/ml, regardless of CD4 + T-cell count (37). Strongly associated with baseline viral load levels is treatment efficacy and response to therapy. HIV RNA levels greater than 150,000 copies/ml correspond to 1.5 times increased likelihood of treatment failure which is the ability to decrease the viral load to less than 50 copies/ml (37).

Variations in viral load between males and females were also clearly noticeable. In congruence with several studies (38-41), we found higher proportions of HIV RNA viral load $\geq 50,000$ copies/ml in males (59.3\% [16/27], 95\% Cl: 38.8-77.61) than females (45.3\% [63/139], 95\% Cl: 36.9$54 \cdot 0)$. Consistent with results from our descriptive analysis which indicate lower proportions of females with HIV RNA viral load $\geq 50,000$ copies/ml than males, model estimation results also confirm significant independent associations for both age and sex. Increasing age was positively associated with new infections ( $P<0.001$, model 1 ), although the correlation with age was abated in controlled models (model $2 \& 3$ ). Irrespective of viral load levels, female adolescents were 2.3 times $(P<0.001$, model 1$)$ at increased risk of HIV seroconversion in relation to males. These differentials are substantively documented in previous studies highlighting up to 50\% lower HIV RNA viral load and higher CD4 + Tcell counts in HIV-1 infected women than men soon after seroconversion (39, 40, 42-44). However, other studies confirm attenuation of the sex effect in advanced stages of infection $(41,45)$. Moreover, increasing age at seroconversion has been associated with increased risk of speedy immunologic deterioration and high virologic replication $(35,37)$. Touloumi and colleagues verified that older age at the time of HIV antibody

Page $12 / 19$ 
seroconversion was associated with shortened period to AIDS indicated by steepest (most negative) HIV RNA level and CD4 + T-cell slopes in the younger population groups like adolescents (46).

Extensive loss of mucosal CD4 + T cells occurs in the early stages of acute HIV infection, once this biomarker of immunologic potential falls below 500 cells $/ \mathrm{mm} 3$, much of the immune reserve is wrecked and infected persons become susceptible to opportunistic infections. In this study, we found that $62 \%$ (95\% Cl: $54 \cdot 65-69 \cdot 46)$ of recently HIV infected adolescents had CD $4+$ T-cell count below 500 cells/mm3 with half of these young people aged 15-24 years diagnosed with CD4 + T-cell count of 479 cells/mm3 (95\% Cl: 445-513.6) from the normal functional range of between 500 cells $/ \mathrm{mm}^{3}$ and 1500 cells $/ \mathrm{mm}^{3}$. As the primary target of HIV, depletion of CD $4+\mathrm{T}$-cells acutely constrains the host response capacity. HIV infects activated cells, causing the T-cells directed against the virus to be at greatest risk of infection $(35,47)$. From the point of acquisition to AIDS, disease progresses has formerly been linked to baseline CD4 + T-cell count, HIV RNA levels and a number of other determinants including age and sex $(48,49)$. In corroboration, our analysis finds that $63 \%$ of males aged $15-24$ with CD $4+T-$ cell counts $\leq 500$ cells $/ \mathrm{mm}^{3}$ coincided with $59 \cdot 3 \%$ of them having HIV RNA $\geq 50,000$ copies/ml compared with $61 \%$ and $45 \cdot 3 \%$ respectively, of their female equivalents. Additionally, $64 \cdot 5 \%$ and $57 \cdot 1 \%$ of adolescents aged 20-24 and 15-19 years separately, had CD4 + T-cell count below 500 cells $/ \mathrm{mm}^{3}$. These results imply that a large proportion of adolescents testing positive for HIV, especially males, already have their immune system potency far deteriorated. In line with our findings, Bosch and colleagues found that younger age was a significant independent predictor of greater CD4 + T-cell count (42). Other studies have verified that age at seroconversion and HIV RNA level are associated with the CD4 + T-cell count at baseline and its subsequent slope to the ultimate disease syndrome $(36,46,47)$. Further estimates highlight that within two years of contracting HIV, older individuals and those with the highest HIV RNA levels during early infection experience the most severe depletion of CD4 + T-cell s (43).

Co-infection, particularly with HBV, was one factor associated with incidence of HIV in young people. We identified that $2 \%(95 \% \mathrm{Cl}$ : $0 \cdot 41-5 \cdot 70)$ of new HIV infections in the population 15-24 years also tested positive for HBV. In our confounder-adjusted models, HBV - positive adolescents were 8.6 times $(P<0.001$, model 2$)$ at increased risk of new infection relative to HBV - negative adolescents in the same age stratum. Because $\mathrm{HBV}$ is more infectious and adolescence is a period of increased sexuality, most infections have been found to occur in adolescents and young adults (50). Our findings align with several studies in Africa that have established increased Hepatitis B virus vulnerability in HIV-positive persons (51-55). Clinically, HBV belongs to a variety of heterologous viruses that have been shown to enhance HIV replication (36). Therefore, individuals who are HIV-positive and also test positive for HBeAg are more likely to transmit both viruses $(25,50)$. The HIV- HBV co-infection augments risk of morbidity, antiretroviral therapy-related hepatotoxicity and mortality beyond those caused by either infection alone (56, 57). Similar to HBV results, syphilis co-infection was also estimated at $2 \%$. Adolescents who tested positive for syphilis antibodies were 1.2 times $(P<0 \cdot 84$, model 2$)$ more likely to contract HIV even though correlation was not statistically significant. Both HBV and syphilis co-infections were observed to occur at HIV RNA > 10,000 copies/ml RNA suggesting increased susceptibility during acute infection. Syphilis has been associated with high-risk sexual behaviour, increasingly prevalent in adolescence (58) and reported to be one of the more frequently occurring infection among in HIV infected people (54).

\section{Conclusion}

Our study suggests new HIV infections in adolescents are first diagnosed at high HIV RNA and low CD4 + T-cell count increasing the risk of transmission and likelihood of treatment failure with reduced ability to achieve viral suppression. Sex differentials highlight disproportionate susceptibility of female adolescents to infection than males. Additionally, confounder-adjusted models ascertained that adolescents who were married or cohabiting, were positive for hepatitis B virus, were rural residents and had attained higher than secondary education emerged as the strongest correlates of new infections. While the epidemic continues to spread within the adolescent population, HIV transmission and acquisition will become more complex and greater proportions of adolescents will be infected by their regular partners, especially rural ones. Which implies an increasing need for interventions targeted at stable partnerships, intensified public health campaigns specific for rural adolescents and population segmented preventive health services. However, adolescents being the less prioritized population group in health response and routinely overlooked in national plans, the advent of the COVID-19 pandemic poses a major threat to reversing minimal preventive gains so far achieved in adolescent HIV prevention.

\section{Abbreviations}

ART Antiretroviral Therapy

CD4 T-Cell CD4 T Lymphocyte

HIV Human immunodeficiency Virus

HIV RNA HIV Ribonucleic acid 
HBTC HIV Home-Based Testing and Counselling

HBV Hepatitis B Virus

STARHS Serologic Testing Algorithm for Recent HIV Seroconversion

ZAMPHIA Zambia Population-based HIV Impact Assessment

\section{Declarations}

\section{Authors' Contribution}

TNM and NM developed the manuscript concept. TNM and NM sourced data, re-coded data, conducted statistical analysis and interpreted analysis results. TNM drafted initial manuscript. XZ, SK, CG, XQ, KS and JW reviewed methods, analysis results and interpretation. All authors edited and approved the final content of the manuscript before submission.

\section{Data sharing}

Data used in this article are available to bona fide researchers on request from Zambia's Ministry of Health (www.moh.gov.zm) through email (info@moh.gov.zm).

\section{Declaration of interests}

All authors declare no competing interests.

\section{Data sharing}

Data used in this article are available to bona fide researchers on request from Zambia's Ministry of Health (www.moh.gov.zm) through email (info@moh.gov.zm).

\section{Funding}

No funding was received to produce the manuscript.

\section{Authors' contributions}

TNM and NM developed the manuscript concept. TNM and NM sourced data, re-coded data, conducted statistical analysis and interpreted analysis results. TNM drafted initial manuscript. XZ, SK, CG, XQ and KS reviewed methods, analysis results and interpretation. All authors edited and approved the final content of the manuscript before submission.

\section{Ethics declaration}

Since this study was based on secondary individual de-identified dataset, no ethical approval was required. However, ethical approval for the protocol for ZAMPHIA Population-based survey on which the study is based was obtained from the Tropical Diseases Research Centre (TDRC) Zambia (FWA00003729), the Centers for Disease Control and Prevention (CDC), Columbia University IRB (FWA \# 00002636), and WESTAT (FWA \# 00005551).

\section{Consent for publication}

Not applicable

\section{Acknowledgements}

Not applicable

\section{References}

1. Ferrand RA, Bandason T, Musvaire P, Larke N, Nathoo K, Mujuru H, et al. Causes of acute hospitalization in adolescence: Burden and spectrum of HIV-related morbidity in a country with an early-onset and severe HIV epidemic: A prospective survey. PLoS Med. 2010;7(2):1-4.

2. UNAIDS. Ending the AIDS epidemic for adolescents, with adolescents A practical guide to meaningfully engage adolescents in the AIDS response. 2016. 
3. UNICEF. UNICEF. (2017) HIV Statistical Tables - Global and Regional Trend [Internet]. 2017 [cited 2020 May 3]. Available from: https://data.unicef.org/topic/hivaids/global-regional-trends/.

4. WHO. HIV and Youth [Internet]. WHO

WHO. HIV and Youth [Internet]. WHO. World Health O. 2017 [cited 2020 May 3]. Available from:

https://www.who.int/maternal_child_adolescent/topics/adolescence/hiv/en/.

5. Ministry of Health (Zambia). Zambia Population-Based HIV Impact Assessment (ZAMPHIA) 2016: Final Report [Internet]. Lusaka; 2019. Available from: http://phia.icap.columbia.edu/wp-content/uploads/2016/09/ZAMBIA-Factsheet.FIN_.pdf.

6. PEPFAR. FACT SHEET The epidemic is becoming controlled in several key African countries. 2017.

7. https://dx.plos.10.1371/journal.pmed.1000227

Gray GE. Adolescent HIV-Cause for Concern in Southern Africa. PLoS Med [Internet]. 2010 Feb 2 [cited 2020 Mar 15];7(2):e1000227. Available from: https://dx.plos.org/10.1371/journal.pmed.1000227.

8. Shisana O, Rehle TM, Simbayi LC, Zuma K, Jooste S. South African National HIV. Prevalence, Incidence, Behaviour and Communication Survey, 2008: A Turning Tide Among Teenagers? Medicine (Baltimore) [Internet]. 2009 [cited 2020 Mar 15]; Available from: https://www.hsrcpress.ac.za/books/south-african-national-hiv-prevalence-incidence-behaviour-and-communication-survey-2008.

9. Zambia Statistics Agency. Zambia Demographic and Health Survey 2018 [Internet]. 2020 [cited 2020 Mar 19]. Available from: www.DHSprogram.com.

10. Barradas DT, et al. Zambia Population-Based Hiv Impact Assessment - Factsheet. J Int AIDS Soc [Internet]. 2017;Vol. 20(December 2016):35-6. Available from: http://phia.icap.columbia.edu/wp-content/uploads/2016/09/ZAMBIA-Factsheet.FIN_.pdf.

11. UNAIDS. UNAIDS calls for greater urgency as global gains slow and countries show mixed results towards 2020 HIV targets | UNAIDS. 2019;1-4. Available from:

https://www.unaids.org/en/resources/presscentre/pressreleaseandstatementarchive/2019/july/20190716_PR_UNAIDS_global_report_2019.

12. Bakaki P, Kayita J, Moura Machado JE, Coulter JBS, Tindyebwa D, Ndugwa CM, et al. Epidemiologic and clinical features of HIV-infected and HIV-uninfected ugandan children younger than 18 months. J Acquir Immune Defic Syndr. 2001 Sep 1;28(1):35-42.

13. Grant AD, Djomand G, Smets P, Kadio A, Coulibaly M, Kakou A, et al. Profound immunosuppression across the spectrum of opportunistic disease among hospitalized HIV-infected adults in Abidjan, Cote d'Ivoire. Aids. 1997;11(11):1357-64.

14. 10.1186/1824-7288-37-29

Ogunbosi BO, Oladokun RE, Brown BJ, Osinusi KI. Prevalence and clinical pattern of paediatric HIV infection at the University College Hospital, Ibadan, Nigeria: A prospective cross-sectional study. Ital J Pediatr [Internet]. 2011 Jun 16 [cited 2020 Mar 18];37(1):29. Available from: http://ijponline.biomedcentral.com/articles/10.1186/1824-7288-37-29.

15. Sawyer S, Azzopardi P, Wickremarathne D, Patton G. The age of adolescence. Lancet Child Adolesc Heal. 2018;2:223-8.

16. Viner RM, Ozer EM, Denny S, Marmot M, Resnick M, Fatusi A, et al. Adolescence and the social determinants of health. Lancet [Internet]. 2012;379:1641-52. [cited 2020 Mar 18 ;... Available from: www.thelancet.com.

17. Patton GC, Olsson CA, Skirbekk V, Saffery R, Wlodek ME, Azzopardi PS, et al. Adolescence and the next generation. Nature. 2018 Feb 21;554(7693):458-66.

18. Patlak CS, Blasberg RG, Fenstermacher JD. Graphical evaluation of blood-to-brain transfer constants from multiple-time uptake data. J Cereb Blood Flow Metab. 1983;3(1):1-7.

19. Selik RM, Linley L. Viral loads within 6 weeks after diagnosis of HIV infection in early and later stages: Observational study using national surveillance data. J Med Internet Res. 2018;20(11):1-12.

20. 20 .

21. UNAIDS. Methods for estimating HIV incidence. 2010.

22. Le Vu S, Pillonel J, Semaille C, Bernillon P, Le Strat Y, Meyer L, et al. Principles and uses of HIV incidence estimation from recent infection testing-a review. Vol. 13, Euro surveillance: bulletin européen sur les maladies transmissibles = European communicable disease bulletin. 2008.

23. Mocroft A, Furrer HJ, Miro JM, Reiss P, Mussini C, Kirk O, et al. The incidence of AIDS-defining illnesses at a current CD4 count $\geq 200$ cells/ $\mu$ L in the post-combination antiretroviral therapy era. Clin Infect Dis [Internet]. 2013 Oct [cited 2020 Mar 23];57(7):1038-47. Available from: http://www.ncbi.nlm.nih.gov/pubmed/23921881.

24. Murray JS, Elashoff MR, lacono-Connors LC, Cvetkovich TA, Struble KA. The use of plasma HIV RNA as a study endpoint in efficacy trials of antiretroviral drugs. AIDS [Internet]. 1999 May 7 [cited 2020 Mar 23];13(7):797-804. Available from: http://www.ncbi.nlm.nih.gov/pubmed/10357378.

25. Highleyman L. Hepatitis B, [Internet] aidsmap. 2017 [cited 2020 Apr 26]. Available from: http://www.aidsmap.com/about-hiv/hepatitis-b. 
26. Salado-Rasmussen K. Syphilis and HIV co-infection: Epidemiology, treatment and molecular typing of Treponema pallidum. Dan Med J. 2015 Dec 1;62(12).

27. Williams R. Models for Count Outcomes. 2015;1-25.

28. StataCorp LLC. Stata version 14·2. College Station. 2016.

29. $10.1080 / 09540121.2020 .1739214$

Mwaba K, Mannell J, Burgess R, Sherr L. Uptake of HIV testing among 15-19-year-old adolescents in Zambia. AIDS Care - Psychol SocioMedical Asp AIDS/HIV [Internet]. 2020 May 13 [cited 2020 Jul 7];32(sup2):183-92. Available from:

https://www.tandfonline.com/doi/abs/10.1080/09540121.2020.1739214.

30. Denison JA, Mccauley AP, Dunnett-Dagg WA, Lungu N, Sweat MD. The HIV testing experiences of adolescents in Ndola, Zambia: Do families and friends matter? AIDS Care - Psychol Socio-Medical Asp AIDS/HIV. 2008 Mar;20(1):101-5.

31. WHO

WHO. Adolescent. HIV testing and counselling: a review of the literature. 2013 [cited 2020 Jul 7]; Available from: https://www.ncbi.nlm.nih.gov/books/NBK217943/.

32. Alcorn K. Viral load critical in heterosexual transmission | aidsmap [Internet]. NAM AIDSMAP. 2001 [cited 2020 Apr 16]. Available from: http://www.aidsmap.com/news/mar-2001/viral-load-critical-heterosexual-transmission.

33. Guy R, Gold J, García Calleja JM, Kim AA, Parekh B, Busch M, et al. Accuracy of serological assays for detection of recent infection with HIV and estimation of population incidence: a systematic review [Internet]. Vol. 9, The Lancet Infectious Diseases. 2009 [cited 2020 Apr 29]. Available from: .

34. Ghys PD, Kufa E, George MV. Measuring trends in prevalence and incidence of HIV infection in countries with generalised epidemics. Vol. 82, Sexually Transmitted Infections. BMJ Publishing Group; 2006. p. i52.

35. Langford SE, Ananworanich J, Cooper DA. Predictors of disease progression in HIV infection: a review. 2007 [cited 2020 Mar 18]; Available from: http://www.aidsrestherapy.com/content/4/1/11.

36. Pantaleo G, Fauci A. Immunopathogenesis of HIV infection. Indian J Med Res. 2005 Apr;121(4):240-55.

37. Pirrone V, Libon DJ, Sell C, Lerner CA, Nonnemacher MR, Wigdahl B. Impact of age on markers of HIV-1 disease. Vol. 8, Future Virology. NIH Public Access; 2013. p. 81-101.

38. Addo MM, Altfeld M. Sex-Based Differences in HIV Type 1 Pathogenesis. 2014 [cited 2020 Apr 29]; Available from: https://academic.oup.com/jid/article-abstract/209/suppl_3/S86/2192880.

39. Farzadegan H, Hoover DR, Astemborski J, Lyles CM, Margolick JB, Markham RB, et al. Sex differences in HIV-1 viral load and progression to AIDS. Lancet. 1998 Nov;7(9139):1510-4. 352(.

40. Griesbeck M, Scully E, Altfeld M. Sex and gender differences in HIV-1 infection. Clin Sci. 2016 Aug 1;130(16):1435-51.

41. 10.1056/NEJM200103083441003

Sterling TR, Vlahov D, Astemborski J, Hoover DR, Margolick JB, Quinn TC. Initial Plasma HIV-1 RNA Levels and Progression to AIDS in Women and Men. N Engl J Med [Internet]. 2001 Mar 8 [cited 2020 Apr 30];344(10):720-5. Available from: http://www.nejm.org/doi/abs/10.1056/NEJM200103083441003.

42. Bosch RJ, Bennett K, Collier AC, Zackin R, Benson CA. Pretreatment Factors Associated With 3-Year (144-Week) Virologic and Immunologic Responses to Potent Antiretroviral Therapy. JAIDS J Acquir Immune Defic Syndr [Internet]. 2007 Mar [cited 2020 Apr 30];44(3):268-77. Available from: http://journals.Iww.com/00126334-200703010-00004.

43. Touloumi G, Pantazis N, Babiker AG, Walker SA, Katsarou O, Karafoulidou A, et al. Differences in HIV RNA levels before the initiation of antiretroviral therapy among 1864 individuals with known HIV-1 seroconversion dates. AIDS. 2004 Aug;20(12):1697-705. 18(.

44. Anastos K, Gange SJ, Lau B, Weiser B, Detels R, Giorgi JV, et al. Association of Race and Gender With HIV 1 RNA Levels and Immunologic Progression. J Acquir Immune Defic Syndr. 2000.

45. Odorizzi PM, Wherry EJ. An interferon paradox. Vol. 340, Science. American Association for the Advancement of Science; 2013. p. $155-6$.

46. Touloumi G, Hatzakis A, Rosenberg PS, O'Brien TR, Goedert JJ. Effects of age at seroconversion and baseline HIV RNA level on the loss of CD4 + cells among persons with hemophilia. AIDS. 1998 Sep;10(13):1691-7. 12(.

47. Stebbing J, Gazzard B, Douek DC. Mechanisms of Disease - Where Does HIV Live? N Engl J Med [Internet]. 2004 [cited 2020 Apr 30 ]; Available from: www.nejm.org.

48. Maini M, Gilson R, Chavda N. al. et. Reference ranges and sources of variability of CD4 counts in HIV-seronegative women and men. Genitourin Med. 1996;72:27-31.

49. Moroni M. Sex differences in HIV-1 viral load and progression to AIDS. Lancet [Internet]. 1999 Feb 13 [cited 2020 Apr 30];353(9152):589-90. Available from: https://linkinghub.elsevier.com/retrieve/pii/S0140673605756464. 
50. Hoffmann CJ, Thio CL. Clinical implications of HIV and hepatitis B co-infection in Asia and Africa. Vol. 7, Lancet Infectious Diseases. Elsevier; 2007. p. 402-9.

51. Chonco FM, Rangiah S. Susceptibility to hepatitis B infection, hepatitis B/HIV co-infections and hepatitis B immunity in HIV-positive patients starting HAART in Durban, South Africa. South African Fam Pract. 2019 Apr 5;61(2):65-8.

52. King J, Hagemeister DT. Hepatitis B co-infection in HIV-infected patients receiving antiretroviral therapy at the TC Newman Anti Retroviral Treatment Clinic in Paarl, Western Cape. Vol. 17, Southern African Journal of HIV Medicine. South African Medical Association; 2016.

53. Jong E, Conradie F, Berhanu R, Black A, John M-A, Meintjes G, et al. Consensus statement: Management of drug-induced liver injury in HIVpositive patients treated for TB. South Afr J HIV Med. 2013 May;20(3):113-9. 14(.

54. Matee MIN, Magesa PM, Lyamuya EF. Seroprevalence of human immunodeficiency virus, hepatitis B and C viruses and syphilis infections among blood donors at the Muhimbili National Hospital in Dar Es Salaam, Tanzania. BMC Public Health. 2006 Jan 30;6.

55. Sutcliffe S, Taha TE, Kumwenda NI, Taylor E, Liomba GN. HIV-1 prevalence and herpes simplex virus 2, hepatitis C virus, and hepatitis B virus infections among male workers at a sugar estate in Malawi. J Acquir Immune Defic Syndr. 2002 Sep 1;31(1):90-7.

56. 10.1056/NEJMp1201796 Kourtis AP, Bulterys M, Hu DJ, Jamieson DJ. HIV-HBV Coinfection - A Global Challenge. N Engl J Med [Internet]. 2012 May 10 [cited 2020 Apr 26];366(19):1749-52. Available from: http://www.nejm.org/doi/abs/10.1056/NEJMp1201796.

57. Tumpa SI, Mamun AA. HIV/HBV Co-infection - A Global Challenge. Med Sci. 2015;14(04):HIV/HBV Co-infection-A Global Challenge.

58. Shilaih M, Marzel A, Braun DL, Scherrer AU, Kovari H, Young J, et al. Factors associated with syphilis incidence in the HIV-infected in the era of highly active antiretrovirals. Medicine (Baltimore) [Internet]. 2017 Jan [cited 2020 Apr 26];96(2):e5849. Available from: http://journals.Iww.com/00005792-201701130-00033.

\section{Figures}

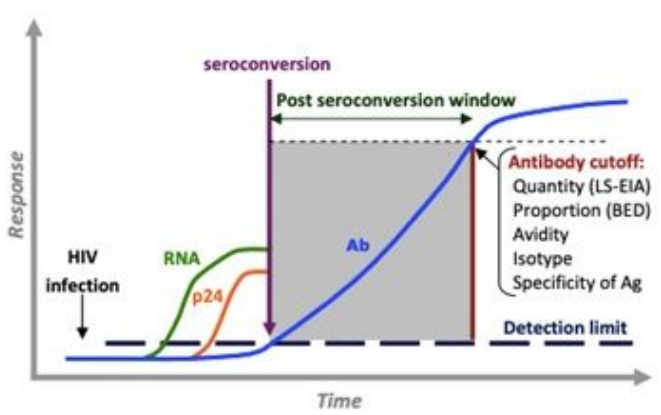

Figure 1

STARHS biomarker detection schema used in estimating new HIV infections 
Figure 2a.

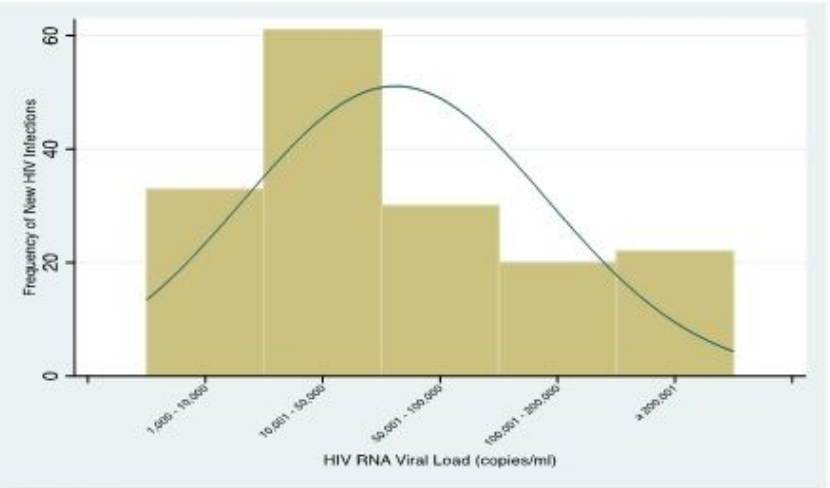

Figure: $\mathbf{2 b}$.

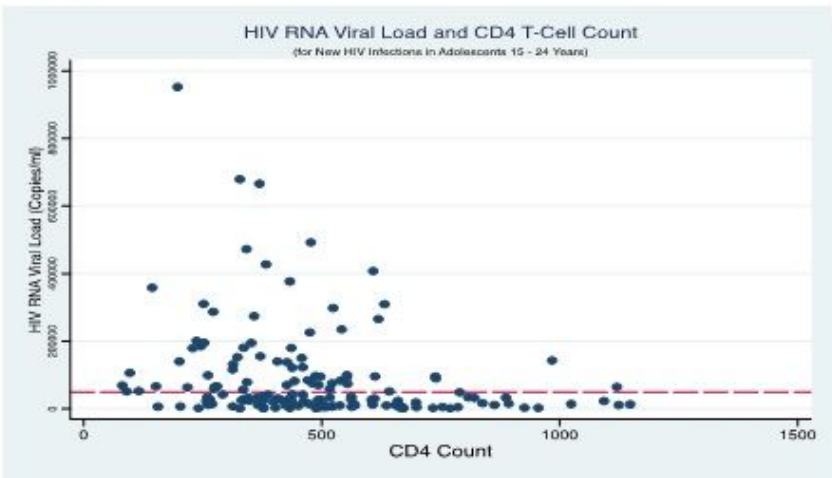

Figure 2

Viral Load and CD4 T-Cell Count for New HIV Infection in Adolescents

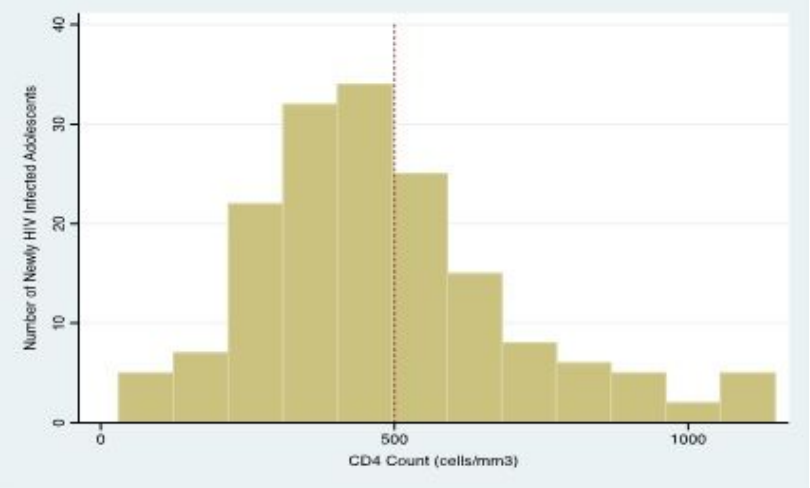

Figure 3

CD4 T-Cell Count of Newly Infected Adolescents
Figure 2c.

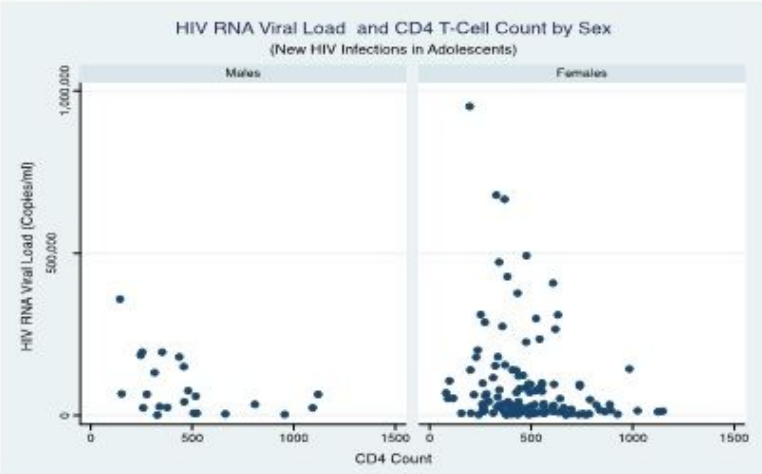

Figure 2d.

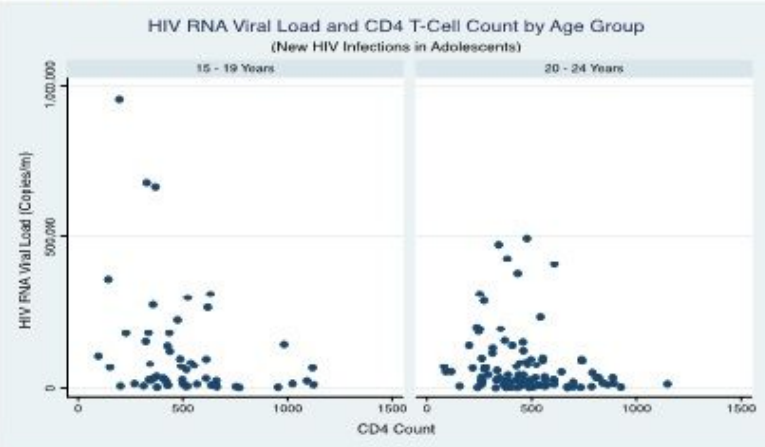


Figure: $4 a$.

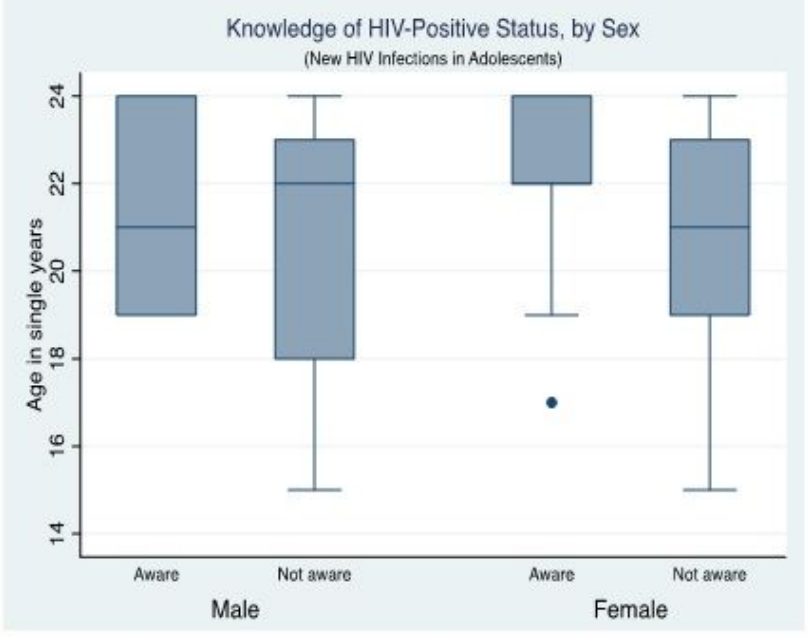

Figure: $4 b$.

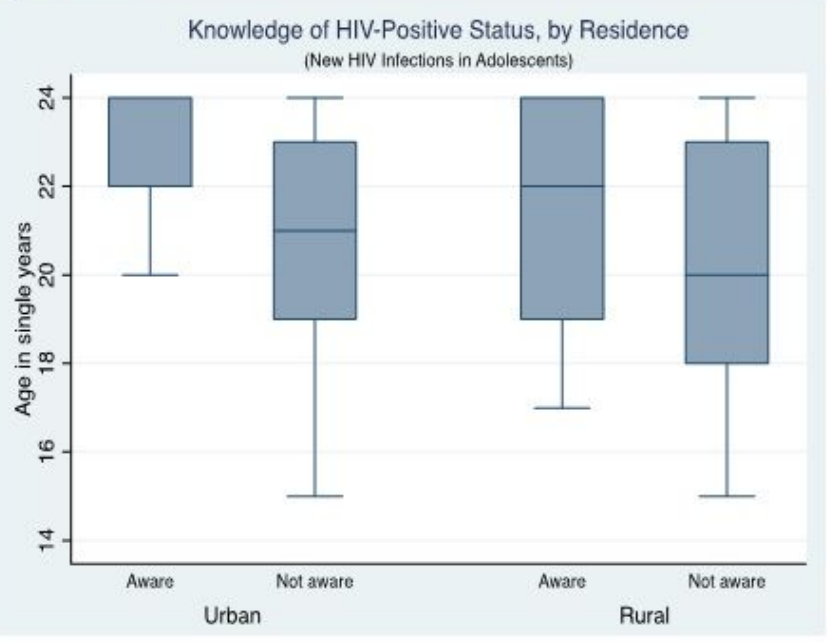

\section{Figure 4}

Figure 4 